\title{
Utilização das Plataformas Web e Ginga-NCL no Desenvolvimento de Aplicações de EAD adaptadas para TV Digital
}

\author{
Maria Lúcia Kroeff Barbosa, PPGEDU - UFRGS, malukroeff@ yahoo.com.br \\ André Schulz, Instituto de Informática - UFRGS, afertschulz@ gmail.com \\ Alexandro Bordignon, Instituto de Informática - UFRGS, abordignon@ inf.ufrgs.br \\ Valter Roesler, Instituto de Informática - UFRGS, roesler@inf.ufrgs.br
}

\section{RESUMO}

Diversas pesquisas estão sendo realizadas em relação ao uso da televisão digital como uma ferramenta de $\mathrm{EaD}$. Aplicativos direcionados para educação, saúde e entretenimento são comuns, entretanto, a grande maioria não conta com o apoio da transmissão dos mesmos através da Transport Stream de alguma emissora específica de televisão. Para executar o aplicativo, o usuário deve utilizar um pendrive ligado ao Terminal de Acesso. Por outro lado, estão surgindo tecnologias de televisão com navegador web embutido, criando duas possibilidades: a) desenvolver um aplicativo NCL/Lua/Java ligado a um pendrive na televisão; b) desenvolver um aplicativo baseado em HTML que acessa um servidor web remoto. Este artigo objetiva analisar ambas as estratégias de desenvolvimento da interação com o usuário e a TV Digital. Para tanto, foram desenvolvidos dois aplicativos em ambas plataformas a fim de melhor comparar as abordagens.

\begin{abstract}
A lot of research is performed nowadays related to the use of digital TV as a distance learning tool. Entertaining and Health applications are usual, however, the great majority does not count with a broadcaster to send them through their Transport Stream. To play the application, the user has to run it through a pendrive connected in the set-top box. On the other side, there are new TV technologies with a web browser embedded, creating two possibilities: a) run the application developed in NCL/Lua/Java in a pendrive connected to a set-top box; b) run an HTML application accessing a remote web server. This paper compares both user/TV interaction strategies. To reach this goal, we developed two applications in both platforms in order to better compare them.
\end{abstract}

\section{INTRODUÇÃO}

Segundo Franco (2003), alguns autores defendem que a garantia do sucesso da Educação a Distância está na motivação do aluno, pois ela proporcionaria a aprendizagem. Sabe-se, também, que o processo educacional não é um processo solitário e que não depende exclusivamente do aluno, mas das relações cognitivas e das sociais. $O$ processo de aprendizagem, para ser emancipador do sujeito aprendiz, necessita ser construtor de conhecimento, e esse processo de construção de conhecimento supõe a construção de estratégias operatórias, o que dificilmente ocorre com o sujeito isoladamente.

As novas tecnologias da informação e da comunicação potencializam essas possibilidades de aprendizagem e, conforme a mídia escolhida para suportar as interações em um curso a distância, tem-se maior ou menor qualidade nas mesmas. Ou seja, nenhuma delas 
impossibilita as interações, mas algumas as otimizam. Se a educação a distância pode ajudar a redimensionar nosso espaço e nosso tempo no ensinar/aprender, o que, em si já é uma grande vantagem, o desafio passa a ser otimizar a interação (Franco \& Behar, 2000).

Pode-se dizer que a tecnologia de TV digital no Brasil, desde seu surgimento em 2006, criou uma expectativa muito grande em relação ao desenvolvimento de aplicações interativas capazes de oferecer uma série de serviços úteis e novas experiências aos telespectadores. O SBTVD (Sistema Brasileiro de TV Digital) definiu o middleware Ginga, definindo os padrões a serem usados no desenvolvimento de aplicativos para essa plataforma.

Com a possibilidade de oferecer interatividade ao cidadão via televisão, muitas pesquisas foram efetuadas visando prestar serviços úteis ao mesmo, aproveitando o fato de que o número de aparelhos de televisão instalados nas residências é vasto, atingindo praticamente a totalidade da população brasileira. Oliveira et al. (2006), atenta para o fato de que a tecnologia da TV Digital interativa trará grandes mudanças ao meio televisivo, hábitos de consumo, ambientes de aprendizagem e ao modo como os indivíduos passarão a se relacionar com essa nova mídia, uma vez que podem utilizá-la para acesso à internet, compras online, home-bank, entre outros. Portanto, esse meio de comunicação poderá possibilitar às pessoas que não tem acesso a computadores terem algum contato com alguns sistemas que se assemelham com os aplicados a eles. Entretanto, para que o aplicativo funcione automaticamente na TV digital, é necessário que ele seja transmitido por uma emissora de televisão através da Transport Stream, e para que isso aconteça, esse aplicativo deve estar alinhado com o modelo de negócios da emissora.

Muitas vezes, entretanto, são criados aplicativos que ainda não estão alinhados com o modelo de negócios de uma emissora de televisão. Tais aplicativos exigem a utilização de um pendrive no Terminal de Acesso da Televisão Digital, sendo executados de forma manual. Exemplos de pesquisas e projetos onde é necessário inserir um pendrive no Terminal de Acesso são muitos, como o TV Digital Social, da Dataprev ${ }^{1}$, que está numa jornada para a disponibilização do aplicativo para a população via emissoras de televisão, o projeto $\mathrm{OBAA}^{2}$, que definiu um novo padrão de metadados educacionais, assim como diretrizes para o desenvolvimento de objetos de aprendizagem interoperáveis entre Web, TV digital e dispositivos móveis (BORDIGNON, 2009).

Em paralelo a essa tendência de crescimento da televisão digital interativa, está acontecendo outra tendência atualmente, que é o crescimento de televisões que permitem execução de aplicativos na Internet, oferecendo uma série de possibilidades, como acesso ao Facebook, Youtube, Skype, Wikipedia, Picasa, entre outros. Entre essas plataformas de TV destacam-se o Google $\mathrm{TV}^{3}$, Boxee ${ }^{4}$, Apple $\mathrm{TV}^{5}$, Microsoft Media Room ${ }^{6}$, OpenTV ${ }^{7}$, e também iniciativas de convênios entre fabricantes de televisores e sites de Internet, como a tecnologia Netcast, da linha Infinita da $\mathrm{LG}^{8}$ e da linha Smart TV da SAMSUNG ${ }^{1}$.

\footnotetext{
${ }^{1}$ http://portal.dataprev.gov.br/2010/07/23/ dataprev-apresenta-tv-digital-social-em-porto-alegre/

${ }^{2}$ http://www.portalobaa.org/

${ }^{3} \mathrm{http}: / / \mathrm{www}$. google.com/tv

${ }^{4} \mathrm{http}: / / \mathrm{www}$.boxee.tv/

${ }^{5} \mathrm{http}: / / \mathrm{www}$. apple.com/appletv/

${ }^{6} \mathrm{http}: / / \mathrm{www} . \mathrm{microsoft} . \mathrm{com} / \mathrm{mediaroom} /$

${ }^{7}$ http://www.opentv.com/

${ }^{8} \mathrm{http}: / / \mathrm{www}$.lginfinita.com.br/

V. $9 \mathrm{~N}^{\mathrm{o}}$ 2, dezembro, 2011
} 
Sendo assim, o presente artigo está organizado da seguinte forma: a seção 2 apresenta alguns trabalhos relacionados. A seção 3 descreve a plataforma do SBTVD, enquanto a seção 4 descreve a plataforma Web. A seção 5 mostra as aplicações desenvolvidas. A seção 6 explica as decisões tomadas para implementar as soluções e na seção 7 são feitas as considerações finais.

\section{TRABALHOS RELACIONADOS}

Vale et al. (2008) discutem as potencialidades e limitações do middleware Ginga-NCL no que diz respeito ao desenvolvimento de aplicações voltadas para a área da educação a distância. Além disso, abordam o panorama do desenvolvimento de aplicações voltadas para a educação e sua evolução de acordo com o surgimento de novas tecnologias, desde os computadores pessoais até o novo paradigma da televisão digital. Para tanto, desenvolveram algumas aplicações utilizando NCL e Lua que foram executadas em um Set-Top-Box comercial com suporte a Ginga-NCL e transmitidos em laboratório através da ferramenta WM-PlayTV, responsável pela geração e modulação do Transport Stream (TS) contendo as aplicações interativas.

Oliveira et al. (2009) destaca a importância de implementar aplicativos de telemedicina no ambiente de TV digital brasileiro, propondo um sistema que aprimora o middleware Ginga, onde é adicionado um suporte a serviços de home care através da integração de diversos dispositivos de hardware e novos componentes de software.

Oliveira, Queiroz Neto e Maeta (2007) colocam que uma das maiores preocupações dos desenvolvedores está em produzir interfaces que permitam aos usuários uma utilização eficiente, efetiva e satisfatória dos recursos disponíveis na TV Digital. Com isso, aprofundaram seus estudos sobre os conceitos de usabilidade e sua relação com as interfaces gráficas interativas utilizadas na TV Digital.

\section{MIDDLEWARE GINGA}

O middleware Ginga foi padronizado para a televisão digital brasileira, onde dois ambientes de programação estão disponíveis (ABNT, 2008): o declarativo, baseado na linguagem NCL (Ginga-NCL), e o procedural, que utiliza uma máquina virtual Java (Ginga-J).

\subsection{Ambiente Declarativo}

O SBTVD escolheu o NCL (Nested Context Language) como linguagem declarativa. A NCL tem uma separação mais acurada entre o conteúdo e a estrutura, não definindo nenhum objeto de mídia em si. Em vez disso, ela define a cola que prende os objetos de mídia em apresentações multimídia. Como linguagem de cola, ela não restringe ou prescreve os tipos de conteúdo dos objetos de mídia. Assim, é possível ter objetos de imagem, vídeo, áudio, texto, outros códigos declarativos ou imperativos sendo referenciados pelo código NCL. O suporte aos diferentes formatos de mídia, porém, dependerá de exibidores de mídia acoplados ao formatador NCL (exibidor NCL, renderizador de documentos, agente do usuário e player são alguns dos nomes atribuídos ao formatador de documentos) (ABNT, 2008).

\footnotetext{
${ }^{1}$ http://www.samsung.com/br/microsite/smarttv/?cid=Digitas_Samsung_Google_SmartTV_SmartTV V. $9 \mathrm{~N}^{\mathrm{o}}$ 2, dezembro, 2011
} 
Embora a linguagem declarativa seja a NCL, como linguagem de cola ela suporta mídias no formato XHTML, sendo que a implementação de um player para esse formato é obrigatória, tendo também como objetivo garantir a interoperabilidade com os demais sistemas (ABNT, 2008). Porém isso torna obrigatório apenas um pequeno conjunto de tags HTML básicas para formatação do documento, o que é muito limitado tendo em vista a criação de aplicações e serviços para televisão digital.

Em relação à linguagem de script do ambiente declarativo, o SBTVD escolheu a linguagem Lua. Lua é uma linguagem de programação funcional e imperativa, procedural, pequena e leve, projetada para expandir aplicações em geral, para ser usada como linguagem extensível e para ser embarcada em softwares complexos (ABNT, 2008). Lua combina programação procedural com poderosas construções para descrição de dados, baseadas em tabelas associativas e semântica extensível. É tipada dinamicamente, interpretada a partir de bytecodes, e tem gerenciamento automático de memória com coleta de lixo. Essas características fazem de Lua uma linguagem ideal para configuração, automação (scripting) e prototipagem rápida, característica de grande importância para as aplicações de TV, cujo desenvolvimento rápido é fundamental. Com seu núcleo pequeno e totalmente ANSI C, ela é facilmente embarcável, onde seu interpretador é uma biblioteca $\mathrm{C}$, embarcável em várias linguagens, entre elas $\mathrm{C} / \mathrm{C}++$ (linguagem da implementação de referência do Ginga-NCL) e Java (linguagem do ambiente imperativo Ginga).

Uma das limitações do ambiente declarativo é que o ECMAScript (ECMA, 2009), é apenas opcional no SBTVD (ABNT, 2008). Dessa forma, a linguagem JavaScript (que é uma das implementações do padrão ECMAScript), muito utilizada na programação Web para computadores pessoais e dispositivos móveis, depende da iniciativa dos fabricantes para incluí-la nos set-top boxes. Porém, nos terminais disponíveis atualmente, o que se tem visto é o não suporte ao JavaScript.

\subsection{Ambiente Procedural}

O Ginga-J é o subsistema lógico com linguagem imperativa do sistema Ginga, baseado na linguagem de programação Java. Um componente-chave do ambiente de aplicação procedural é a máquina de execução do conteúdo procedural, composta por uma máquina virtual Java (ABNT, 2008).

Embora inicialmente a escolha natural fosse pelo GEM (DVB, 2011), uma solução de middleware já adotada por diversos padrões de TV digital do mundo, o Fórum SBTVD, numa parceria com a SUN Microsystems, optou por criar uma nova especificação, o JavaDTV. Essa substitui bibliotecas que poderiam incorrer em royalties futuramente, como o HAVi Level 2 UI para construção da interface e o DAVIC para gerenciamento de recursos escassos (DVB, 2011), por equivalentes funcionais, além de especificar funcionalidades adicionais aos outros padrões, como o suporte a múltiplos dispositivos.

Um limitante hoje para programação em Java para TV digital é a não existência de um ambiente de simulação e testes do Ginga-J e nem set-top boxes comerciais disponíveis com esse ambiente. Portanto, neste momento, a programação para TV digital ainda permanece limitada pela utilização das linguagens NCL e Lua, conforme poderá ser visto nas aplicações implementadas, que serão descritas mais adiante.

Outra limitação consiste no fato de que a inclusão do ambiente procedural nos dispositivos de recepção de TV digital móvel (recepção one-seg) não é obrigatória (ABNT NBR 15604, 
2008). Dessa forma, aplicativos desenvolvidos para o ambiente procedural de TV digital poderão não funcionar nos aparelhos móveis de recepção (one-seg).

\section{PLATAFORMA WEB}

O HTML (Hypertext Markup Language) é a linguagem adotada pela Web para descrever os diversos tipos de documentos que apresentam as formas possíveis de conteúdo a quem navega. Sua principal característica é o uso de tags para indicar elementos de estrutura visual nos documentos (layout), possuindo diversos tipos de elementos para esse fim, tais como imagens, tabelas, marcadores de parágrafos e criação de divisões hierárquicas.

O XHTML é uma linguagem de marcação que tem a mesma abrangência de expressão do HTML, mas segue as leis da sintaxe XML (Extensible Markup Language). Dessa forma, pode-se utilizar o XHTML como a interseção do HTML com o XML em muitos aspectos. Como eles precisam estar bem formatados, documentos XHTML corretos permitem seu processamento automático utilizando ferramentas XML padrão, ao contrário do HTML, o qual requer um parser relativamente complexo, lento e específico.

Já o CSS (W3C CSS, 2011) é utilizado localmente pelo web browser para definir cores, fontes, layout e outros aspectos da apresentação de documentos HTML. Foi projetado primeiramente para possibilitar a separação do conteúdo do documento (escrito em HTML ou XHTML) da sua apresentação (escrito em CSS). Essa separação melhora a acessibilidade ao conteúdo, fornecendo maior flexibilidade e controle da especificação das características de apresentação, reduzindo a complexidade e repetição do conteúdo.

Para criação de lógica dinâmica, matemática e procedural no lado do cliente, usam-se linguagens de Script, onde o padrão existente hoje é o ECMA-262 (ECMAScript Release 3), baseado em diversas tecnologias originárias, sendo mais conhecidos o JavaScript (Netscape) e JScript (Microsoft).

Recentemente a especificação da linguagem HTML foi atualizada, sendo chamada de HTML-5 (W3C, 2011), uma versão sucessora das versões HTML 4.01 e XHTML 1.1 que introduz novas marcações e APIs. Entre esses novos recursos, destacam-se os relacionados à multimídia, que têm o objetivo de facilitar a inclusão e gerenciamento de vídeo, áudio e elementos gráficos na Web, sem recorrer a APIs e plugins proprietários como o Flash, por exemplo.

No momento, o HTML5 ainda se encontra em padronização e a versão atual dos navegadores Web implementam alguns dos novos recursos do HTML5. Da mesma forma, apenas os dispositivos móveis mais modernos já estão prevendo suporte a algumas funcionalidades do HTML5.

\section{APLICAÇÕES IMPLEMENTADAS}

Seguindo a previsão de que, num futuro próximo, aplicativos para TV digital estarão disponíveis ao telespectador proporcionando serviços úteis, contendo cada vez mais interfaces ricas e funcionalidades, foram desenvolvidos dois aplicativos distintos que seguem essa tendência: um oferece serviços de telemedicina integrado com orientações educacionais na área da saúde e o outro é uma solução mais clássica de ensino a distância (EAD). Foram feitas duas versões para cada aplicativo, uma na plataforma GingaNCL/LUA e a outra baseada em HTML na plataforma PC. As implementações em cada plataforma são diferentes, porém seguem uma única especificação, ou seja, objetivam 
oferecer exatamente as mesmas funcionalidades da aplicação em questão. Sendo assim, foi possível destacar as capacidades de cada plataforma frente ao mesmo objetivo, buscando utilizar os melhores recursos de cada uma. As seções a seguir descrevem os dois aplicativos desenvolvidos.

\subsection{Aplicação de Telemedicina TV-Care}

A fim de contribuir para promover a autonomia e a melhoria da qualidade de vida das pessoas idosas, deve-se ter em mente a importância do desenvolvimento de um programa educacional que atenda às especificidades desse público em uma modalidade que se dará a distância. Em função de todas as características citadas anteriormente, fica evidente que propostas educativas para esse público alvo exigem metodologias especiais para os processos de ensino-aprendizagem. No âmbito das tecnologias da informação, por exemplo, geralmente se observa que as pessoas da terceira idade têm medo do novo/desconhecido e, quase sempre, precisam de incentivo para iniciar o processo. Machado e Behar (2011) recomendam as seguintes questões que devem ser analisadas e respondidas para que sejam possibilitadas metodologias mais adequadas para esse contexto: quais as tecnologias/recursos que devem ser utilizados com idosos? Qual a melhor estratégia de ensino? Como o professor poderia mediar essa interação (idoso/tecnologia/aprendizagem)?

Pensando sobre esses aspectos, o TV-Care oferece serviços de gerenciamento em saúde através do uso de um aparelho de televisão ligado a um dispositivo computacional, que pode ser um notebook ligado na televisão ou um Terminal de Acesso de TV digital. Através da utilização do sistema, busca-se prover melhora na autonomia do idoso ativo através de três diretrizes básicas resumidas na Figura 1: a) monitoramento de sua saúde via sensores de sinais vitais e comunicação com uma central médica; b) conscientização de seu estado de saúde e re-educação física e alimentar; c) maior interação social com equipe de saúde, amigos, familiares e outros usuários do sistema através de ferramentas de comunicação.

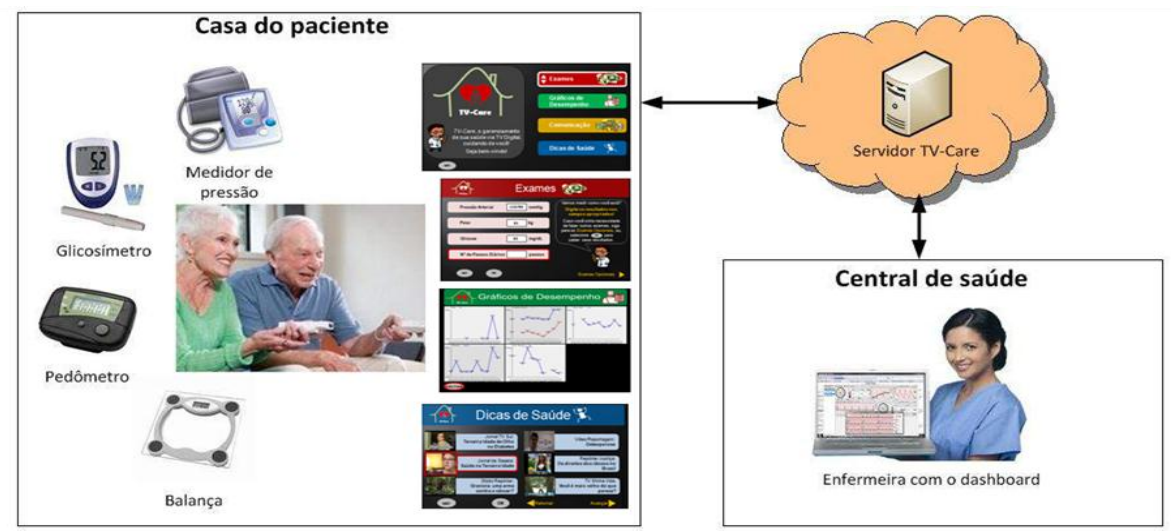

Figura 1 - Arquitetura do TV-Care

Pode-se perceber que à disposição do idoso existe um ou mais sensores de sinais vitais, tais como glicosímetro e medidor de pressão arterial. Além disso, existe um pedômetro cuja função é medir o número de passos e uma balança para verificação do peso corporal. $\mathrm{O}$ terminal de acesso da TV digital ou outro equipamento, como o notebook ou um celular $3 \mathrm{G}$, recebe os dados e os envia para uma central médica, de forma que os sinais do idoso possam ser monitorados e, caso algum parâmetro esteja fora do esperado, alguém entra em contato com o mesmo ou envia uma ambulância imediatamente. Esses sensores podem 
receber os sinais de forma automática (via Bluetooth, por exemplo), ou de forma manual, através da digitação dos valores via controle remoto ou outra forma de interação.

A interface de interação com a televisão provê diversas possibilidades ao idoso, como as "Dicas de Saúde", que atualmente consistem em aproximadamente 150 vídeos. Além disso, ele pode verificar seus "Gráficos de Desempenho" ao longo do tempo, percebendo a variação do estado dos seus sinais vitais. O sistema foi pensado para atender principalmente ao público da terceira idade, por isso a interação do usuário com a aplicação deve ser simples e pode ocorrer através do controle remoto da TV. A Figura 2 mostra a tela inicial do TV-Care.

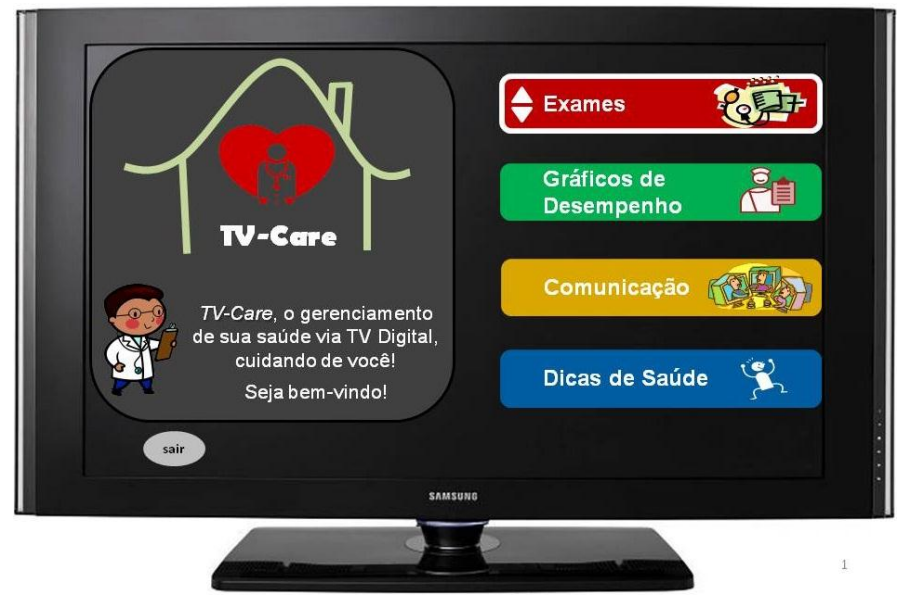

Figura 2 - Menu principal do TV-Care

Como observado na figura, o aplicativo ganha características próprias do ambiente de TV digital interativa, apresentando vários requisitos que devem atender principalmente a questões de apresentação apropriada do conteúdo na tela, à navegação clara e simples nos menus e ao tratamento dos eventos disparados pelo usuário através do controle remoto. Além disso, o aplicativo deve oferecer suporte a uma série de protocolos de rede, para se comunicar com: a) o sistema de cadastro e armazenamento dos dados dos usuários; b) o servidor de vídeo sob demanda; c) o servidor de videoconferência na web.

Para que a aplicação seja compatível com qualquer Terminal de Acesso que suporte o SBTVD, foi desenvolvida uma versão inicial do sistema nas linguagens NCL e Lua, para ser corretamente executada no middleware Ginga, mais precisamente na sua parte declarativa que já possui implementação real no mercado, chamada Ginga-NCL/Lua.

A intensa interatividade demandada pelo TV-Care e sua grande quantidade de funcionalidades existentes são facilmente encontradas em programas desenvolvidos para serem executados em computadores pessoais, mas no momento em que tais funcionalidades são transferidas para o cenário dos aplicativos de TV digital, no qual o Ginga faz parte e onde o suporte tecnológico é reduzido, aparecem limitações dos mais variados tipos, abordadas na seção 6 .

Buscando fazer um aproveitamento com outra tecnologia existente, foi desenvolvida uma versão do TV-Care com as mesmas funcionalidades do aplicativo em Ginga-NCL/Lua para ser executada em PC, feita através de HTML, Javascript e outros recursos de programação Web. Para que essa versão em PC não perdesse a característica de aplicativo de TV digital, foi utilizado um netbook com um sistema operacional convencional, um browser com 
suporte aos recursos de script e um programa que gerencia eventos de controle remoto recebidos por uma interface de infravermelho. Sendo assim, foi possível identificar e descrever as diferenças entre as plataformas, evidenciadas nos seguintes aspectos ou características do aplicativo TV-Care: navegação nas telas e menus, entrada de dados usando o controle remoto, exibição e execução de áudio e vídeo, suporte aos recursos de videoconferência, gerenciamento dos dados gerados pelo usuário e comunicação da aplicação com o servidor de informações dos usuários, tais como o desenvolvimento de um sistema de alertas sobre os horários de realização de cada exame e apresentação da funcionalidade de recomendação de vídeos educativos apresentados de acordo com os resultados dos exames.

\subsection{Aplicação de Educação a Distância}

O "Viva Saudável" é um curso sobre a melhoria da qualidade de vida através da alimentação saudável e da prática regular de atividade física. Foi pensado para jovens entre 12 e 15 anos, mas pode ser cursado por outras faixas etárias que tenham interesse no assunto, pois não possui censura. Está dividido em três módulos, sendo que o estudo apresentado neste artigo aborda o módulo 1, cuja principal função é relembrar alguns conceitos e experiências vividas, motivando os alunos à reflexão sobre seus hábitos alimentares e sobre a importância do exercício físico. Prevê atividades como quiz, vídeo e testes para verificação de massa corporal. Ele foi implementado em NCL/Lua, Web e também dispositivos móveis.

A interface do índice é apresentada na Figura 3, e pode-se observar as diversas lições implementadas no curso, inclusive atividades "para fazer" e questionários para testar o conhecimento do aluno.

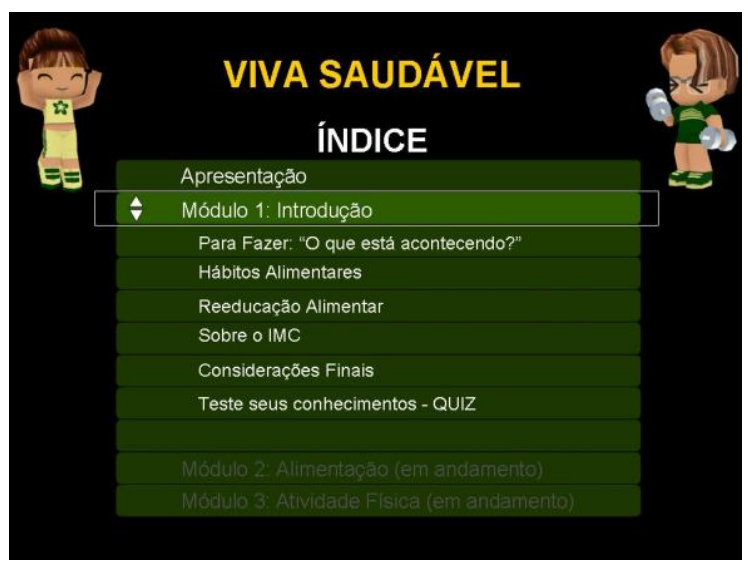

Figura 3 - Menu principal do aplicativo de EAD

\section{SOLUÇÕES ADOTADAS}

A análise comparativa dos aplicativos implementados aborda aspectos qualitativos da experiência de uso das linguagens envolvidas e da capacidade de execução das funcionalidades das aplicações nas plataformas. Sendo assim, a linguagem preferida para o aplicativo de EAD "Viva Saudável" foi a linguagem NCL/LUA, apesar de não ser possível executar as funções de reprodução dos vídeos na plataforma Ginga-NCL/Lua real. A simplicidade da interação do "Viva Saudável" e seus vídeos de caráter educativo são mais facilmente implementados nessa plataforma. 
No caso do aplicativo TV-Care, a adoção da plataforma Ginga-NCL/Lua se mostrou inadequada frente às dificuldades de se implementar as funcionalidades dos protocolos de streamings adicionais, da comunicação e sincronização com o servidor de dados e da limitação de recursos para realização de videoconferências. Por isso, foi preferido o uso de um netbook executando uma aplicação baseada em HTML com recursos Web para comunicação de dados, streaming de conteúdo multimídia e recursos para videoconferências.

\subsection{Pontos positivos da solução Ginga-NCL}

As principais vantagens da linguagem NCL estão no sincronismo das mídias e na capacidade de alterar suas representacões visuais em tempo de execução. $O$ uso dessa plataforma se mostrou vantajosa na implementação de aplicativos interativos mais simples como é o caso do curso "Viva Saudável". Um exemplo disso é a apresentação de indicações visuais ou textos em vídeos educativos.

\subsection{Pontos positivos da solução Web em PC}

Por ser a plataforma que engloba todas as tecnologias que a Internet proporciona, fica evidente que os requisitos envolvendo comunicação com servidores remotos no aplicativo TV-Care são melhores resolvidos através dessa plataforma. Entre suas principais vantagens estão: videoconferência, streaming de conteúdo multimídia, comunicação segura com servidores remotos e uso de Web services através de JSF (JSF, 2011). Além disso, destacase a atual capacidade de armazenamento do PC em relação aos Terminais de Acesso de TV.

\section{CONSIDERAÇÕES FINAIS}

Este artigo apresentou características de duas tendências tecnológicas que permitem a comunicação e a interação do usuário com a televisão: uma delas é o uso do middleware de TV Digital Ginga, e a outra é o uso de um navegador web, prevendo sua inclusão nos últimos televisores.

Considerando que a mesma solução seja disponibilizada como produto final em diversas plataformas possíveis, não somente no Ginga, considera-se que soluções baseadas em HTML juntamente com recursos Web aproveitariam melhor as tecnologias já existentes para produzir conteúdo educacional aos usuários.

Sendo assim, os aplicativos desenvolvidos e implentados neste estudo se candidatam a funcionar em qualquer plataforma com o mínimo de suporte a HTML e com alguns recursos adicionais, como Java e plugins para exibição de vídeos. Porém, isso não significa que a plataforma Ginga de TV digital não seja capaz de suportar esses aplicativos, pois a utilização de sistemas de navegação (browsers) pelo middleware está na norma do SBTVD e páginas contendo aplicações baseadas em HTML estão previstas para serem executadas pela máquina do NCL. Portanto, pode-se concluir que um modelo possível é o Ginga-NCL executando a parte de sincronismo de mídias, que é seu forte, e para interações com suporte HTML, o Ginga pode utilizar um navegador web embutido.

\section{REFERÊNCIAS}

ABNT - Associação Brasileira de Normas Técnicas. ABNT NBR 15604. Televisão digital terrestre - Receptores. 2008. Disponível em: http://www.dtv.org.br/download/ptbr/ABNTNBR15604_2007Vc_2008.pdf Acesso em julho de 2011. 
BORDIGNON, A. et. al. Mechanisms for interoperable content production among Web, Digital TV and Mobiles. In: Proceedings of WCCE 2009: IX IFIP WORLD CONFERENCE ON COMPUTERS IN EDUCATION. July, 2009. Bento Gonçalves, Brazil.

DVB. Introduction to MHP \& GEM. 2008. Disponível em: http://www.mhp.org/introduction.htm Acesso em abril de 2011.

ECMA - Standard ECMA-262, "ECMAScript Language Specification - Edition 5". 2009. Disponível em: http://www.ecma-international.org/publications/files/ECMA-ST/Ecma262.pdf Acessado em julho de 2010.

FRANCO, S. R. K. Algumas reflexões sobre educação à distância.Textual, Porto Alegre, v. 1. n. 2, p. 6-11, ago. 2003.

FRANCO, S. R. K. BEHAR, P. Fundamentos epistemológicos da educação a distância. In: Curso de formação em educação a distância. Módulo1. Unirede e Universidade Federal do Paraná, 2000.

LuaRocks. Disponível em: http://luarocks.org/repositories/rocks Acesso em maio de 2011.

MACHADO, L.; BEHAR, P. A. Formação de professores para cursos online com o público idoso: um desafio para a educação. Disponível em: http://api.ning.com/files/9aVVzK2W9ixCIv5fgfgzLh74yYXGerPIctHjOW4rmE8RvGvoTr iEEPPtIcZcvE51vgQLPhrbrq4mvf096Z3*bRmCZ9wRQ7/FORMAODEPROFESSORESP ARACURSOSONLINECOMO.pdf Acesso em junho de 2011.

OLIVEIRA, J.; WAISMAN, T.; SILVA, F.; HORBE, B.; MAIA, O.; MORENGHI, F. Tlearningin amazon: The interactive education project. ACM Computing Reviews. 2006.

OLIVEIRA, L. S.; QUEIROZ-NETO, J. P.; MAETA, S. M. A Usabilidade em Interfaces interativas no desenvolvimento de aplicativos para a TV Digital. In: II Congresso de Pesquisa e Inovação da Rede Norte Nordeste de Educação Tecnológica. João Pessoa/PB, 2007.

OLIVEIRA, M.; CUNHA, P. R. F.; SANTOS, M. E. S.; Bezerra, J.C.C. Implementing home care application in Brazilian Digital TV. In: Information Infrastructure Symposium, 2009. GIIS '09. Global, vol., no., pp.1-7, 23-26 June 2009.

VALE, D. T.; DINIZ, R.; MELONI, L.G.P. Desenvolvimento de Aplicações Educacionais para Televisão Digital: Proposta de Mídias em Flash. Revista de Radiofusão. São Paulo. V. 3. n. 3. p. 143-150. ago. 2009.

W3C. HTML5. W3C Working Draft. 2011. Disponível em: http://www.w3.org/TR/html5/ Acesso em abril de 2011. 
11

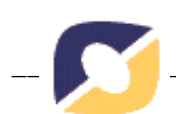

JSF - Java Server Faces. Disponível em:

http://www.oracle.com/technetwork/java/javaee/javaserverfaces-139869.html Acesso em maio de 2011.

W3C. Cascading Style Sheets. 2011. Disponível em: http://www.w3.org/2011/05/css-pr Acesso em abril de 2011. 